\title{
LE GOUT DES JEUNES POUSSES: ATTICUS, BRUTUS, OCTAVE
}

\section{Yasmina Benferhat*}

Key words: Politique; jeunesse; Rome; Brutus; Octave; Atticus (Politics; youth; Rome; Brutus; Octavius; Atticus)

Brutus ... Octave ... Quel est le point commun? L'un est né vers 85 avant JC, l'autre est né vingt ans plus tard en 63 avant JC, appartenant ainsi à une tout autre génération. Un premier point commun pourrait être César qui favorisa la carrière de l'un comme de l'autre, qui fut tué par l'un et vengé par l'autre. Un second point commun pourrait se trouver dans la plaine de Philippes, là où les soldats de Brutus prirent le camp d'Octave, là où Brutus se suicida après la victoire d'Antoine. Mais c'est le troisième point commun que nous voudrions étudier ici, en la personne de Titus Pomponius Atticus. ${ }^{1}$ Ce chevalier romain des plus discrets se trouva parmi les proches de Brutus, et plus tard dans l'entourage d'Octave devenu Octavien. Né en 110 avant $\mathrm{JC}$, il mourut un an avant Actium. Il avait donc vingt-cinq ans de plus que Brutus et presque cinquante ans de plus qu'Octave. Ses talents étaient multiples: on le voit faire un arbre généalogique de la famille de Brutus, ${ }^{2}$ à la demande de

1 Les biographies d'Atticus ne manquent pas en anglais et en allemand: voir en particulier Leslie (1950) et Perlwitz (1994). Voir également Benferhat 2005: 98-172.

$2 \quad C f$ Nep Att 18 3. Sur les généalogies d'Atticus, préparées en 46-44, voir Marshall 1993: 308310 sur celle de Brutus. L'auteur souligne les aspects politiques de ces ouvrages, tout en les rapprochant du Liber annalis d'Atticus pour ce qui est du travail d'historien.

* $\quad$ Maître de conférence habilitée, Université de Lorraine. 
celui-ci qui savait le goût d'Atticus pour l'histoire. On le voit donner des conseils à Octavien pour rénover un temple ancien tombé en ruine: ${ }^{3}$ toujours cette passion pour l'histoire ...

La question que l'on pourrait se poser pour commencer serait de savoir ce qu'ils pouvaient s'apporter vraiment, ce qu'ils attendaient les uns les autres de leur relation. Rome affectionnait et favorisait des liens entre générations qui permettaient de transmettre des savoirs: pensons à la relation qui existait entre le questeur et son gouverneur de province quand les choses se passaient bien. Le questeur, ${ }^{4}$ jeune homme d'environ trente ans, était comme un fils adoptif pour le consulaire: l'un commençait sa carrière quand l'autre était parvenu au sommet, ne pouvant plus guère espérer que la charge de censeur s'il n'était pas encore rassasié d'honneurs. Il y avait donc comme une sorte de passage de relais entre deux générations. De même, les spécialistes de droit ou les grands orateurs attiraient auprès d'eux des jeunes gens venus se former: Cicéron eut ainsi parmi ses protégés Caelius et Trebatius, après avoir été formé par Mucius Scaevola.

Mais la relation avec Atticus n'était pas de cet ordre-là: il choisit de ne pas faire carrière et de rester dans l'ombre, donc il n'avait pas de savoir officiel - que cela soit d'ordre militaire ou politique ou intellectuel - à transmettre. En revanche, il était extrêmement riche et faisait partie de la haute société romaine avec un carnet d'adresses très solide. Il était donc représentatif d'une certaine Rome et c'est peutêtre dans cette direction qu'il faudra chercher. Inversons le point de vue: que pouvait attendre Atticus de jeunes pousses comme Brutus et Octave? Quel intérêt pouvait-il trouver à les fréquenter? Ils étaient tous les deux promis à un avenir de chef de parti, mais pas forcément le parti qui plaisait le plus à Atticus. Nous verrons donc dans une première partie comment il a soutenu Brutus, puis comment il a dû se faire à Octave: chemin faisant nous reverrons la Rome des dernières années de la République et le terreau de l'ascension fulgurante du futur Auguste.

$$
* * *
$$

Lorsque Brutus naquit, ${ }^{5}$ Atticus n'était pas à Rome, si l'on retient la date de 85 avant JC pour cette naissance. Tous les deux étaient pris dans les tourbillons de la guerre civile qui avait commencé en 88: Atticus avait choisi de partir s'installer à Athènes ${ }^{6}$ après le meurtre du tribun $\mathrm{P}$ Sulpicius Rufus, auquel il était apparenté.

$3 \quad$ Idem 203.

4 Voir Moreau 1989: 37-46. Sur la questure, voir Latte (1936) sur les origines de cette magistrature; voir Harris (1976) pour son histoire sous la République entre 267 (création de cette charge) et 81 (la réforme de Sylla) et Ryan (1996) sur l'âge du questeur.

5 Parmi les nombreux ouvrages consacrés à Brutus, voir en particulier Clarke (1981) et Dettenhofer 1992: 99-119 sur ses jeunes années. Martin a consacré plusieurs études à Brutus, de sa thèse sur la royauté jusqu'à maintenant: nous renvoyons à l'une des dernières qui permettra de trouver la bibliographie complète: voir Martin 2010: 33-49.

6 Sur les raisons de ce séjour (financières, politiques) voir Marshall 1999: 56-68. L'auteur insiste sur les liens financiers d'Atticus avec le monde grec en mettant en avant la figure de Lucullus auquel était lié Caecilius, l'oncle d'Atticus. 
Orphelin de père, il laissait à Rome sa mère Caecilia et sa soeur Pomponia. La mère de Brutus, Servilia, ${ }^{7}$ avait épousé un partisan de Marius, Marcus Junius Brutus, ${ }^{8}$ tribun de la plèbe en 83 que l'on croise dans le Pro Quinctio, et qui fut assassiné en 77 par Pompée au moment du soulèvement organisé par Lépide. ${ }^{9}$

Non seulement Brutus perdit ainsi son père alors qu'il n'avait pas dix ans, mais il semble bien qu'il devint enfant de proscrit avec l'impossibilité de faire carrière ensuite: $F$ Hinard considère que $M$ Junius Brutus n'avait pas déposé les armes contre Sylla ${ }^{10}$ mais que parti en Gaule il y entretint une insurrection à la façon d'un Sertorius en Espagne, et qu'il se rallia à Lépide par la suite. C'est donc en tant que personne inscrite sur la liste des proscrits qu'il aurait été mis à mort par Pompée quand les Syllaniens parvinrent à lui mettre la main dessus. Cela expliquerait en tout cas l'adoption du jeune Brutus par le frère de sa mère, Q Servilius Caepio, dont il prit le nom: c'était un moyen de lui permettre de faire le cursus honorum.

C'est le nom sous lequel il est connu en 59 avant JC lorsqu'il est accusé de conspirer contre Pompée sous le consulat de César: Cicéron utilise néanmoins les deux patronymes Caepio hic Brutus, dans une lettre à Atticus ${ }^{11}$ qui était peut-être déjà intéressé par le sort de cette jeune pousse jugée prometteuse dans le camp des Optimates. C'est en effet à ce titre que son nom fut cité par un dénonciateur avec celui d'autres jeunes gens du même parti: Curion, Paulus, Lentulus. On leur reprochait de prendre le parti de Bibulus contre son collègue.

Ses débuts dans le cursus honorum datent de la fin des années 50: auparavant il avait été dans le staff de Caton, le demi-frère de sa mère Servilia, lorsque celui-ci fut envoyé à Chypre ${ }^{12}$ par Clodius en 58 avant JC. C'était un bon moyen de l'éloigner de Rome après cette affaire de conspiration et de l'aider à s'enrichir. Brutus avait commencé à gagner de l'argent par des prêts, y compris à Quintus Cicéron, ${ }^{13}$ mais surtout dans le monde grec. Il fut questeur en 53 avant JC en Cilicie, et épousa à la même époque Claudia, fille d'Ap Claudius Pulcher, ${ }^{14}$ le célèbre consul de 54, gouverneur de Cilicie en 53-52 et censeur de 51.

Il est très clairement alors compté comme la relève à venir dans le camp des Optimates auquel s'est rallié Pompée par son propre mariage avec Cornelia et l'union de son fils aîné avec la belle-soeur de Brutus, autre fille d'Appius. Cela transparaît en

7 RE 101. Voir Dettenhofer 1992: 777-779 sur le rôle politique de Servilia \& Förtsch 1935: 88-94 et 104-10. Syme (1967) a consacré plusieurs analyses à Servilia également (voir index).

8 Magistrates of the Roman Republic 263.

9 Voir Keaveney 1982: 111-139.

10 Voir Hinard 1985: 361-362.

$11 C f$ Nep Att 2242.

$12 C f$ Plut Brut 3. Voir Badian (1965) sur les détails de cette mission.

13 Cf Cic $Q F r 13$.

$14 R E$ 297. Sur ce personnage voir Constant (1921) et plus récemment David 1992: 825-826; Deniaux 1993: 396-397. On trouve dans Syme 1967: 46sqq, quelques notations acidulées sur Appius. L'arbre généalogique des Claudii se trouve dans Syme 1986: VII. 
particulier dans une lettre ${ }^{15}$ de Cicéron à celui-ci en 51. La mère de Brutus, Servilia, avait pourtant essayé de le mettre sous la protection de César en le fiançant à Julie, ${ }^{16}$ mais celle-ci fut finalement donnée en mariage à Pompée.

La correspondance de Cicéron lors de son proconsulat en Cilicie en 51 permet de voir qu'Atticus est alors déjà proche de Brutus: Cicéron le charge de transmettre ses plaintes au gendre d'Appius, qu'il appelle noster Brutus. ${ }^{17}$ Le possessif sert clairement à souligner que l'affection portée à Brutus par Atticus est partagée par Cicéron, et que le jeune homme lui est cher. En revanche, le tuus utilisé par Cicéron dans une lettre postérieure semble indiquer déjà une prise de distance: "Ego tui Bruti rem sic ago ut suam ipse non ageret." ${ }^{18}$ Cette interprétation nous semble confirmée par une critique implicite du caractère difficile de Brutus: "Faciam tamen satis tibi quidem, cui difficilius est quam ipsi; sed certe satis faciam utrique."

C'est que Brutus avait prêté de l'argent au roi Ariobarzane, placé sous la protection de Cicéron ${ }^{19}$ en tant que proconsul de Cilicie, et il comptait bien le récupérer, en se montrant sans doute assez pressant. Cette mentalité ne posait aucun problème à Atticus qui faisait la même chose. ${ }^{20}$ Les deux hommes géraient leurs finances d'une manière semblable, et ce dans la même zone d'influence au sens large, à savoir le monde grec: la nuance est que l'on connaît surtout les relations d'affaires d'Atticus avec Athènes, Sicyone dans le Péloponnèse et la région de Buthrote, ${ }^{21}$ alors que pour Brutus il s'agissait semble-t-il de régions plus à l'Est, qu'il avait eu l'occasion de découvrir au cours de sa mission à Chypre, sous les ordres de Caton, ensuite pendant sa charge de questeur. Atticus appuya donc les demandes de son protégé quand celuici remit à Cicéron une liste de demandes ${ }^{22}$ mandatorum libellus.

Certes Cicéron revendique sa familiaritas avec Brutus - qu'il appelle encore une fois tuus - devant Cassius, ${ }^{23}$ qui était son beau-frère; certes il essaie de faire plaisir à Atticus en affirmant ne pas aimer le jeune homme moins que lui. Là encore le jeu sur les pronoms personnels est révélateur: "quem non minus amo quam tu, paene dixi quam te." ${ }^{24}$ C'est néanmoins Atticus qui est le protecteur des intérêts de Brutus. On peut imaginer sans trop grand risque d'erreur qu'il s'agissait ici pour

$15 C f$ Cic Fam 34 2: il utilise alors le nom M Brutus.

16 Cf Plut Cés 14 7. Voir Syme 1967: 36.

17 CfCic Att 5176.

18 Cic Att 5184 (20 septembre 51).

19 Voir Braund (1983) sur Cicéron et Ariobarzane III.

20 Sur les finances d'Atticus, on peut consulter Leslie 1950: 21-24 (un ensemble assez bref, où l'auteur s'intéresse surtout à la mise en pratique de l'épicurisme dans la gestion du patrimoine d'Atticus), et surtout Perlwitz 1992: 30-85.

21 Voir Horsfall 1989(b): 60-62.

22 Cic Att 613.

23 Cic Fam 15146.

24 Cic Att 5206. 
Pomponius d'utiliser ses relations afin de soigner ses liens avec un jeune homme appelé à devenir un des grands du parti conservateur.

De fait, Lucullus ${ }^{25}$ était mort en 57; les frères Metelli, ${ }^{26}$ aussi bien Celer (le consul de 60) que Nepos (consul en 57) étaient décédés dans les années 50 aussi; Hortensius ${ }^{27}$ n'était plus que l'ombre de lui-même avant de mourir en 50. Par l'intermédiaire de Servilia, sa mère, Brutus était lié à tous ces hommes, du passé désormais, ce qui lui assurait une légitimité certaine à leur succéder: son oncle Caepio avait épousé Hortensia, et sa tante était devenue la seconde femme de Lucullus. ${ }^{28}$ Il y avait certes encore Appius Claudius Pulcher, Scipion Nasica, qui avait été adopté par Metellus Pius, et Caton le Jeune, autrement dit la génération ${ }^{29}$ née entre 97 et 95 . Mais Appius était couvert de scandales et bien trop imbu de sa noblesse: surtout il avait fonctionné en tandem avec son frère le tribun de la plèbe Clodius pour le plus grand bénéfice de la gens Claudia d'abord. Caton ${ }^{30}$ était jugé trop rigide, en particulier vis-à-vis des chevaliers manieurs d'argent. On comprend le souhait de voir émerger un autre homme pour défendre la cause des Optimates.

Atticus, qui n'était pas aussi neutre en politique qu'on le prétend souvent, ${ }^{31}$ avait choisi, et il protégeait Brutus quitte à se retrouver en pleine contradiction, comme on le voit avec l'affaire de Salamine de Chypre. Cicéron, lors de son proconsulat en Cilicie, fut amené à devoir prendre la défense des habitants de cette cité contre un créancier dénommé Scaptius, qui réclamait deux cents talents et qui avait obtenu du beau-père de Brutus un titre de préfet ainsi que la cavalerie qui allait avec pour maltraiter ses débiteurs. ${ }^{32}$ Faute d'arriver à obtenir quelque chose, cet individu finit par sortir la plus belle carte de son jeu: il travaillait en fait pour le compte de Brutus, ${ }^{33}$ qui n'avait pas jugé opportun de le dire, semble-t-il, même à Atticus.

On voit en filigrane une jeune pousse assez imbue d'elle-même et manifestant pratiquement les mêmes défauts que son beau-père Appius: Cicéron utilise les deux adverbes contumaciter et adroganter ${ }^{34}$ pour décrire la façon dont Brutus lui écrivit

25 Voir la biographie de Van Ooteghem (1959) et plus récent Tröster (2008). De la même génération que Lucullus et Hortensius on doit citer Catulus, mort vers 61.

26 Voir Van Ooteghem (1967) et Skinner 2011: chapitre 5.

27 Voir Gruen 1974: 51-53 et Dyck (2008) sur Hortensius: c'est une étude biographique très complète, et une des plus récentes mises au point.

28 Cf Plut Cat 24 4-5. Harders (2007) considère qu'elle était la nièce de Caton et non la sœur de Servilia: contra RE et Neue Pauly IX 461.

29 Nous ne citons ici que les personnages les plus connus: voir Gruen 1974: 56-59 sur Favonius, Bibulus, Domitius.

30 Voir la biographie de Fehrle (1983) et Gruen 1974: 53-56.

31 Sur cette question très débattue, nous nous permettons de renvoyer le lecteur à notre thèse: Benferhat 2005: 124-164.

32 CfCic Att 52110 en particulier.

33 Cf Cic Att 6 1 6. Sur cette affaire, voir Andreau 2001: 40-43. Voir également Welch 1996: 463466.

34 Idem 7. On retrouve la même critique dans une autre lettre $c f$ Cic Att 637. 
alors. Il est clair néanmoins que celui-ci, choyé par Atticus, le choyait à son tour en comptant sur lui pour faire bouger Cicéron avec lequel il avait manifestement beaucoup moins d'affinités. Atticus alla jusqu'à demander cinquante hommes en armes à Cicéron pour aider Brutus et son homme de paille Scaptius, sans se souvenir apparemment qu'il avait lui-même recommandé à son ami proconsul de veiller à sa réputation en se montrant irréprochable: "Ain tandem, Attice, laudator integritatis et elegantiae nostrae, ausus es hoc ex ore tuo ... inquit Ennius, ut equites Scaptio ad pecuniam cogendam darem me rogare?" 35

Sautons quelques années qui virent le début de la guerre civile et la victoire de César sur Pompée à Pharsale où Brutus combattit du côté des Républicains: c'est dans les années 46-44 que les liens entre Atticus et Brutus sont à nouveau bien attestés. Cette période est particulièrement intéressante parce qu'elle voit Brutus dans une position pour le moins ambiguë: ${ }^{36}$ sa carrière rebondit grâce à César qui lui confie la Gaule à gouverner (en 46-45) puis la préture urbaine en 44, mais il est également devenu le chef de file potentiel de l'opposition puisque tous les chefs du parti des Optimates sont morts.

On comprend assez l'intérêt que pouvait lui porter Atticus; en revanche on ne sait malheureusement pas quelle influence le vieil homme a pu avoir sur son cadet pour le pousser ou non contre César. Relevons cependant que faire l'arbre généalogique de Brutus n'était pas vraiment innocent dans le contexte d'alors. En effet, appuyer les prétentions des Junii à faire remonter les origines de la famille au premier Brutus que Rome connut, ${ }^{37}$ celui qui chassa les Tarquins du pouvoir, c'était entrer dans le jeu de toute une propagande invitant Brutus à chasser lui aussi un tyran. Mais c'est Brutus qui lui demanda ce travail, M Bruti rogatu, selon le biographe d'Atticus. Cicéron, dans son Brutus, les décrit arrivant ensemble chez lui:

Nam cum inambularem in xysto et essem otiosus domi, M. ad me Brutus, ut consueuerat, cum T. Pomponio uenerat, homines cum inter se coniuncti tum mihi ita cari itaque iucundi, ut eorum aspectu omnis quae me angebat de republica angebat cura consederit. ${ }^{38}$

Double Patte et Patachon, en somme ... De fait, Cicéron passe par Atticus pour avoir des nouvelles de Brutus alors gouverneur de Gaule. ${ }^{39}$ L'épisode de la publication de l'éloge de Caton par Brutus invite cependant à nuancer quelque peu ce portrait: on

35 Cic Att 628.

36 Son divorce d'avec Claudia en 45 avant JC put donner l'impression qu'il allait manifester son ralliement complet à la cause de César, mais il se remaria avec Porcia, veuve de Domitius et fille de Caton, ce qui marquait nettement sa volonté de prendre la tête du parti des Républicains ( $c f$ Cic Att 1392 de la mi-juin 45). Sa mère ne s'y trompa pas, qui eut de très mauvaises relations avec sa seconde bru ( $c f$ Cic Att 1322 4).

37 Voir Wiseman 1987: 207.

38 Cic Brut 10. Sur ce traité de Cicéron voir le très récent ouvrage de Aubert-Baillot \& Guérin 2014.

$39 C f$ Cic Att 12273 et 12362 (cf 1237 1). De même Cic Att 13 92. Leslie 1951: 52 suggère que Cicéron ait pu ressentir une certaine jalousie devant les liens entre Atticus et Brutus. 
voit Atticus essayant en vain d'influencer Brutus afin qu'il améliore son oeuvre avec l'aide de Cicéron. Celui-ci ne se fit pas faute de souligner l'attitude désagréable du jeune homme: " $[A d]$ cetera uero tibi quem ad modum rescripsit! Tantum rogat de senatus consulto ut corrigas." ${ }^{40}$

Néanmoins, Atticus continua de jouer ce rôle qui lui seyait si bien de passeur en invitant Cicéron à dédier un ouvrage à Brutus ${ }^{41}$ en le faisant copier par ses esclaves, en poussant Brutus à accepter de bon gré le cadeau même si les choix rhétoriques de Cicéron et les siens étaient aux antipodes. C'était une façon comme une autre, assez délicate et astucieuse, de maintenir et de tisser continuellement des liens entre deux hommes ${ }^{42}$ qui avaient pour seul point commun d'être opposés à un retour éventuel de la monarchie.

Cette proximité de Brutus et d'Atticus est exaltée même dans la biographie de celui-ci. Cornelius Nepos vante la relation exceptionnelle, selon lui, ${ }^{43}$ qui s'était établie entre les deux hommes en particulier après le meurtre de César: "Sic M. Bruto usus est ut nullo ille adulescens aequali familiarius quam hoc sene neque solum eum principem consilii haberet, sed etiam in conuictu." 44 ' $\mathrm{C}$ est le terme adulescens qui doit retenir notre attention: en 44 avant JC quel était l'âge de Brutus? Quarante ans et des poussières ... Autrement dit, il y a ici une exagération du biographe pour mettre en valeur le lien exceptionnel entre ces deux hommes de génération différente et surtout le charme d'Atticus qui opérait sur tous les âges: ${ }^{45}$ Brutus n'était plus un adulescens - terme employé pour les jeunes hommes entre dix-sept et trente ans à Rome - il était un iuvenis. ${ }^{46}$ Mais comme certains ont une éternelle tête de gendre idéal, lui semblait toujours concourir dans la catégorie des jeunes espoirs, sans doute aussi parce que les circonstances ne lui permirent jamais d'aller plus loin.

\section{$* * *$}

Certains jugeront donc que l'expression "jeune pousse" était bien abusive pour Brutus, puisqu'on le voit apparaître sur la scène à presque trente ans déjà. Elle s'appliquait bien mieux à Octave avec ses dix-huit ans tout frais lorsqu'il devint

40 Cic Att 12211.

$41 \quad C f$ Cic Att 13 21a 1.

42 Atticus poussait également à des rencontres entre les deux hommes, cf Cic Att 1339 2, même lorsque Cicéron doutait des intentions réelles de Brutus.

43 La biographie d'Atticus composée par Cornelius Nepos a fait l'objet de plusieurs mises au point soulignant souvent l'importance des choix idéologiques de son auteur au détriment de l'exactitude historique: voir Labate \& Narducci 1981: 127-182; Millar 1988: 40-55 et le commentaire de Horsfall 1989a.

$44 \quad$ Nep Att 82.

45 Nep Att 161.

46 Mentionnons cependant Axelson (1948) qui défend la thèse que les deux termes étaient synonymes, adulescens et iuvenis, et que le second supplanta même le premier à partir de l'époque augustéenne. 
héritier officiel de César au printemps 44. C'est qu'il y a jeunesse et jeunesse $\mathrm{e}^{47}$ en politique à Rome, en fait, comme on peut le voir justement avec ces deux hommes: il y a la jeunesse convenable, celle qui a l'âge du questeur, trente ans au minimum donc, quand on commence à parler d'elle, et puis il y a la jeunesse qui déboule dans le jeu avec toute son insolence à vingt ans, parce que les circonstances sont exceptionnelles - retour de Sylla en Italie pour le jeune Pompée - ou parce qu'elle se veut exceptionnelle. Ce fut le cas de toutes ces jeunes pousses traînant en justice une célébrité: César attaqua Dolabella à vingt-trois ans, Caelius attaqua Antonius Hybrida au même âge, Asinius attaqua Caton à vingt-deux ans.

Octave appartient sans aucun doute possible à cette deuxième catégorie de "jeune pousse": en acceptant l'héritage de César dont il devenait le fils adoptif, il menaçait directement la position de Brutus en tant qu'assassin de César, mais aussi la position de Marc Antoine en tant que successeur de César. En somme on se retrouvait avec la génération de 63 qui s'en prenait à la génération de 85-83. Non seulement il était très jeune, mais en plus il n'appartenait pas au milieu des élites de Rome dont faisaient partie Brutus, Atticus et même Antoine qui était le petit-fils de l'orateur Antonius. Octave était le fils d'un homo nouus, Octavius, et de la nièce de César Atia: ${ }^{48}$ orphelin de père à l'âge de quatre ans, il fut élevé par sa mère et son beau-père Philippus. César, après avoir décidé d'en faire son héritier, soigna son éducation dans le domaine de la guerre en l'envoyant à Apollonie commencer à préparer une expédition chez les Parthes.

Atticus voit arriver Octave à Rome en avril 44, alors qu'il soutient Brutus ${ }^{49}$ qui représente un espoir de paix à ses yeux quand les Césariens sont vus comme une menace dans la crainte qu'ils ne veuillent venger César. Au cours de cette première phase d'observation méfiante, le jeune homme est sous-estimé par tous, sauf probablement par Balbus mais c'est une autre histoire. Il n'est pas aisé de savoir avec certitude ce que pensait Atticus d'Octave: une lettre de Cicéron du 19 avril 44 nous permet néanmoins de savoir qu'il ne lui prédisait pas grand avenir une fois l'héritage de César accepté à cause du risque de conflit avec Antoine. Le passage est corrompu: "Octavius Neapolim uenit XIII Kal.; ibi eum Balbus mane postridie eodemque die mecum in Cumano; illum hereditatem aditurum, sed, ut scribis, $\dot{\rho} \eta \xi \dot{i} \theta \varepsilon \mu \mathrm{n}$ magnam

47 La jeunesse à Rome, sujet complexe s'il en est, a fait l'objet en particulier d'une synthèse de Eyben (1993), spécialiste de la question. On trouve également des réflexions intéressantes sur les âges de la vie à Rome dans Néraudau 1984: 21-61. Le fond du problème était probablement le fossé de dix ans entre prise de la toge virile et âge requis pour la questure pendant lequel il fallait patienter, ou se signaler par le tribunat de la plèbe ou un procès. La question fut justement réglée par Auguste qui remodela le cursus et créa des charges nouvelles pour ces années-là.

48 Sur Atia et l'enfance d'Octave, voir Néraudau 1996: 39-59. Octave fut confié à sa grand-mère Julia, auprès de qui il vécut de 59 à 51 lorsque Julia décéda.

49 Une lettre de Cicéron atteste qu'il est en contact permanent avec Brutus, $c f$ Cic Att 148 2. Et le jeu très ironique des adjectifs possessifs tuus/meus montre la même chose, $c f$ Cic Att 14 10 1: Hoc meus et tuus Brutus egit ... 
cum Antonio." ${ }^{\circ 0}$ Nous suivons ici la lectio de la CUF qui essaie de faire avec ce que laissent à deviner les manuscrits. L'hypothèse rixam timet de D Shackleton-Bailey ne nous séduit pas entièrement à cause de l'introduction du verbe timere, comme si Octave avait peur d'Antoine, ce qui n'est pas sûr. Cicéron qui aime si souvent jouer avec le grec dans ses analyses politiques avec Atticus ${ }^{51}$ pourrait très bien simplement indiquer que son ami prévoit un affrontement sévère entre les deux Césariens.

Quels étaient les motifs de méfiance? Ce n'était pas tant la personnalité d'Octave, mal connue sinon inconnue, et de toute façon décrédibilisé par son jeune âge aux yeux de Cicéron et sans doute d'Atticus: il est systématiquement appelé $p u e r,{ }^{52}$ alors que c'est un adulescens comme le dit très justement un Césarien. Non, c'est que son entourage, dont le fameux Balbus ${ }^{53}$ mais également Matius, ${ }^{54}$ pourrait lui donner l'idée de venger le meurtre de César. On trouve un écho sûr de l'hostilité d'Atticus à l'égard d'Octave lorsque Cicéron fait écho à ses commentaires à propos des premiers pas en public du jeune homme:

De Octaui contione idem sentio quod tu, ludorumque eius apparatus et Matius ac Postumus mihi procuratores non placent; Saserna collega dignus. Sed isti omnes, quem ad modum sentis, non minus otium timent quam nos arma. ${ }^{55}$

La contio fait allusion au discours d'Octave du 7 (ou 8) mai. Les ludi évoqués sont les jeux de la Victoire de César préparés pour la fin juillet 44 . Il faut préciser que d'après Cicéron c'est Atticus qui organisa en sous-main ceux que Brutus alors préteur devait organiser - les ludi Apollinares - pour le même mois. ${ }^{56}$ On le devine également essayant de garantir le talent de Cicéron pour appuyer la cause de Brutus, en vain. ${ }^{57}$

Octave est vu comme un pion, que l'on pourrait gagner à la cause de Brutus, que l'on pourrait opposer à Antoine, indispensable pour régler les problèmes des habitants de Buthrote chers à Atticus, ${ }^{58}$ mais de plus en plus tyrannique:

In Octauiano, ut perspexi, satis ingeni, satis animi, uidebaturque erga nostros ut nos uellemus animatus. Sed quid aetati credendum sit, quid nomini, quid hereditati, quid

50 Cic Att 14103.

51 Voir ainsi Dunkel 2000: 122-129.

$52 C f$, par exemple, Cic Att 14122 et Cic Att 169 et Cic Att 16 11, 6; puerile cf Cic Att 1681 (début novembre 44). Idem Cic Fam 10283.

53 Voir Alföldy 1976: 43-54, et Benferhat 2005: 247-256 sur Balbus. Egalement Roddaz 2010: 133143.

54 Celui-ci fut commissaire aux jeux de la Victoire de César (Victoriae Caesaris) organisés les 20-30 juillet par Octave et célèbres pour le passage d'une comète dans le ciel: voir Néraudeau 2007: 64-66. Matius décrit Octave comme optimae spei adulescens ac dignissimus Caesare, cf Cic Fam 1128 6. Voir Grattarola 1990: 44-47.

55 Cic Att 1523 (18 mai 44).

$56 \quad C f$ Cic Att 15182.

$57 \quad$ Cf Cic Att 1542.

58 Voir Deniaux 1987: 245-254. 


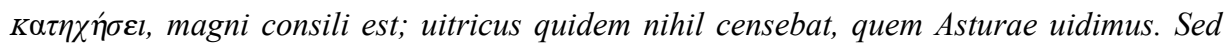
tamen alendus est et, nihil aliud, ab Antonio seiungendus. ${ }^{59}$

Ce que l'on voit ici, c'est une inversion de situation: de même qu'Atticus et Brutus partageaient les mêmes codes qu'un homo novus comme Cicéron ne possédait pas, de même Cicéron est séduit dans le fond par Octave fils d'un homo novus alors qu'Atticus reste méfiant ${ }^{60}$ devant celui qui était un intrus sur tous les plans. Cicéron est aussi sensible à la popularité du jeune homme, en particulier dans les municipes, un monde qui lui était cher.

Une étape suivante voit donc Cicéron choisir de soutenir les entreprises d'Octave, alors qu'Atticus était plus réticent, comme on le voit dans une lettre de la mi-novembre:

Ad ea autem quae scripsisti (tris enim acceperam III Id. a te epistulas), ualde tibi adsentior, si multum possit Octauianus, multo firmius acta tyranni comprobatum iri quam in Telluris, atque id contra Brutum fore ... ${ }^{61}$

Atticus considérait Octave comme un danger beaucoup plus grand qu'Antoine contre les meurtriers de César et attendait donc de voir sa réaction au moment de la fin des fonctions de l'un des complices des Ides de mars, C Servilius Casca, tribun de la plèbe en 44: "Sed ut scribis, certissimum uideo esse discrimen Cascae nostri tribunatum ..."

La suite de la correspondance en 43 ne nous permet pas de savoir clairement quelle fut l'attitude d'Atticus face à Octave: on devine cependant que la stratégie adoptée par Cicéron de soutenir Octave pour éliminer Antoine qu'il considérait comme le plus grand danger, n'était pas partagée par les autres admirateurs des Ides de mars, dont Atticus très probablement: "egregius puer Caesar, de quo spero equidem reliqua." ${ }^{33}$ Dans cette description d'Octave il faut noter surtout le equidem qui marque un certain isolement. Nous ne pouvons que supputer que les doutes exprimés par Brutus en mai 43 à propos d'Octave étaient partagés par Atticus: "Itaque timeo de consulatu, ne Caesar tuus altius se ascendisse putet decretis tuis quam inde, si consul factus sit, descensurum." ${ }^{64}$

La répétition de l'adjectif tuus est assez éclairante: on a un ton de reproche ici, et une mise en garde. Brutus souligne plus loin la crainte que lui inspire l'héritier de César: "quod utinam inspectare posses timorem de illo meum!" Une autre

59 Cic Att 15122 (9 ou 10 juin 44).

60 Leslie 1950: 55 considère que cette méfiance venait peut-être de Brutus qui aurait influencé Atticus.

61 Cic Att 16141.

62 Cic Att 16153.

63 Cic Fam 10283 (à Trebonius, début février 43).

64 Cic $A d B r 14 a 2$. Voir Ortmann (1988) sur la relation triangulaire entre Cicéron, Brutus et Octave, et Willcock (1996) pour un commentaire de la correspondance en 43. 
lettre de Brutus, adressée à Atticus ${ }^{65}$ exprime les mêmes angoisses, mais elle est jugée apocryphe par plusieurs. ${ }^{66} \mathrm{Il}$ est assez évident qu'Atticus lui aussi n'avait pas confiance en Octave et doutait de la stratégie de Cicéron. On sait qu'il aida financièrement Brutus dans sa fuite en lui faisant envoyer d'abord cent mille, puis trois cent mille sesterces comme le rapporte son biographe Cornelius Nepos ${ }^{67}$ avant, il est vrai, de mettre en lumière, l'aide apportée à Fulvia épouse de Marc Antoine. Antoine ou Brutus, de toute façon ce n'était jamais en faveur d'Octave.

Alors que faire de ce passage de la biographie d'Atticus décrivant des liens exceptionnels avec Octave? Reprenons un peu l'extrait qui concerne les fiançailles entre Tibère et la petite-fille d'Atticus:

Quae coniunctio necessitudinem eorum sanxit, familiaritatem reddidit frequentiorem. Quamuis ante haec sponsalia non solum, cum ab urbe abesset, numquam ad suorum quemquam litteras misit, quin Attico mitteret, quid ageret, in primis, quid legeret quibusque in locis et quamdiu esset moraturus, sed etiam, cum esset in urbe et propter infinitas suas occupationes minus saepe quam uellet, Attico frueretur, nullus dies temere intercessit, quo non ad eum scriberet, cum modo aliquid de antiquitate ab eo requireret, modo aliquam quaestionem poeticam ei proponeret, interdum iocans eius uerbosiores eliceret epistulas. ${ }^{68}$

Soulignons tout d'abord que c'est Octave qui fait des efforts pour soigner Atticus, et non l'inverse; le champ lexical également ne plaide pas en faveur d'une grande complicité entre les deux hommes puisque necessitudo renvoie simplement à un lien de parenté, tandis que le comparatif frequentiorem peut laisser supposer que les relations étaient assez limitées alors que la fille d'Atticus ${ }^{69}$ était déjà mariée à Agrippa, principal lieutenant d'Octave.

Il faudrait ensuite se demander quelle période est ici décrite: il ne s'agit certes pas des mois qui précédèrent la mise en place du second Triumvirat, mais bien plutôt des temps qui ont suivi la proscription, quand Atticus survivait à un monde qui n'était plus. Il était désormais obligé de se plier au bon vouloir des maîtres du moment, Antoine et Octave, et la signification politique du mariage de sa fille avec Agrippa n'a peut-être pas été soulignée assez: ${ }^{70}$ ils se sont mariés en 37 , l'année des accords de Tarente.

Permettons-nous une hypothèse: de même que la mise en place du premier triumvirat fut scellée par deux mariages, celui de Pompée avec Julie et celui de César avec Calpurnia, de même après les accords de Brindes scellés par le double mariage

65 Cic $\mathrm{Ad} B r 117$.

66 Voir ainsi J Beaujeu, éditeur de la correspondance, CUF (Paris, 1996), tome XI, 175-182, en particulier 179 pour l'analyse des invraisemblances.

67 Nep Att 96.

68 Nep Att 19 4-20, 1-2.

69 Elle était née probablement vers 51: voir Horsfall 1989a: 84.

70 Voir ainsi Leslie 1951: 60 qui ne s'intéresse guère de toute façon aux années suivant la mort de Cicéron. Voir cependant Roddaz 1984: 80-84 et plus récemment Canas 2012: 155-164. 
d'Antoine et d'Octavie et d'Octave avec Scribonia, le traité de Tarente fut suivi de l'union de la fille d'un partisan d'Antoine avec le bras droit d'Octave. Ce mariage avait un poids politique indéniable et devait contribuer à renforcer, manifester aussi, la volonté d'entente des deux rivaux. ${ }^{71}$ Accessoirement, il confirme qu'Atticus était classé dans les soutiens d'Antoine, qui le sauva de la proscription. Mais aussi il montre la perte d'indépendance d'Atticus puisque les membres de sa famille étaient désormais utilisés comme des pions sur l'échiquier des triumvirs. Lorsque Octave multiplie les signes d'égard, sinon d'affection, à l'égard d'Atticus, il s'agit ni plus ni moins de se mettre dans les traces des disparus, Cicéron et Brutus, pour créer une illusoire continuité avec une période républicaine bien achevée. Atticus et Octave avaient de fait tous deux intérêt à s'entendre, l'un pour sauver ce qui restait à sauver, l'autre pour asseoir son pouvoir.

$* * *$

Que retenir de ces relations entre membres de différentes générations au moment de la fin de la République? On aura compris qu'il y avait plusieurs âges pour apparaître comme la relève en politique: vingt ans, trente ans, quarante ans. A vingt ans on risquait d'être traité de puer en voulant bousculer les autres; à trente ans on avait le profil idéal en entamant le cursus honorum; à quarante ans on pouvait encore faire illusion surtout si des circonstances exceptionnelles, comme une guerre civile, empêchaient d'avancer sa carrière comme à la normale. Octave et Brutus ont offert deux profils différents de jeunes pousses, le moins âgé finissant par l'emporter. D'autre part, le rôle joué par Atticus permet de mesurer la connivence entre membres de l'élite romaine, qu'ils fassent ou non carrière, et le choc que représenta de ce fait l'irruption d'Octave sur la scène. Il fut l'homme de la nouitas, même s'il a cherché ensuite à se rallier ces anciennes élites: Atticus en offre un bon exemple.

\section{Abstract}

Youth in politics might be a default - a lack of experience justifying waiting for your turn - or an advantage, as a promise of renewal and energy. In ancient Rome youth was something more positive than one could expect: the Roman people, if not the Senate, was fond of young leaders like Scipio Nero, whilst Galba was despised for being too old. This paper aims at studying the case of two young men in the Late

71 Contra Potter 1934: 669 qui considère ce mariage comme une tentative d'Antoine pour détacher Agrippa d'Octave, avant que celui-ci ne reprenne la main en fiançant la fille de son lieutenant âgée d'un an au futur Tibère. Cela soulève la question de la marge d'indépendance d'Agrippa par rapport à Octave dans des années de tensions entre celui-ci et Antoine: on peut se demander s'il aurait conclu une alliance déplaisant au fils adoptif de César. Quoi qu'il en soit, qu'Octave ait été favorable ou non, cela ne change rien au fait qu'Atticus devait désormais renoncer à sa neutralité comme à son indépendance en acceptant que sa fille soit mariée en fonction des alliances politiques. 
Republic - Brutus and Octavian - through their relationship with a much older man, Atticus. The first part focuses on Brutus, born approximately $85 \mathrm{BC}$ and belonging to the ancient elite like Atticus, who was considered a promising young leader of the Optimates since his beginnings in $58 \mathrm{BC}$ until his misfortune in 44-43 when he could not manage to apply the murder of Caesar to his own advantage. The second part concerns Octavius, the real young man, born in 63 . He was nineteen years old when he became Caesar's heir, while Brutus was already forty. The vocabulary is revealing: Octavius is called puer by his opponents, but he is an adulescens for the Caesarians, and Brutus is described as an adulescens though already a iuvenis. Atticus, who always helped Brutus, had to change his way: a wedding between Attica and Agrippa, planned by Antony, resulted in him finally belonged to Octavian's party. Octavian, the new man who had won the war, needed a symbol of the old elite and of Republican Rome. Atticus had to save what could be saved.

\section{Bibliographie}

Alföldy, G (1976) Oktavians Aufstieg zur Macht (Munich)

Andreau J (2001) Banque et affaires dans le monde romain (Paris)

Aubert-Baillot, S \& Guérin, C edd (2014) Le Brutus de Cicéron. Rhétorique, politique et histoire culturelle (Leyde)

Axelson B (1948) "Die Synonyme adulescens et iuvenis" in Mélanges de philologie, de litterature et d'histoire anciennes offerts à J. Marouzeau par ses collègues et élèves étrangers (Paris): 7-17

Badian, E (1965) "M Porcius Cato and the annexation and early administration of Cyprus" $J$ of Roman Studies 55: 110-121

Benferhat, Y (2005) Ciues Epicurei. Les épicuriens et l'idée de monarchie à Rome et en Italie de Sylla à Octave (Bruxelles)

Braund, DC (1983) "Cicero and the Greek East. Antiochus I and Ariobarzanes III" Liverpool Classical Monthly 7: 39-40.

Canas, M (2012) “Octavien, Agrippa et Atticus. La place des alliances matrimoniales dans la consolidation de la faction d'un dynaste" in R Baudry \& S Destephen (edd) La société romaine et ses élites. Hommages à Elisabeth Deniaux (Paris): 155-164

Clarke, ML (1981) The Noblest Roman: Marcus Brutus and His Reputation (Londres)

Constant (1921) Un correspondant de Cicéron: Appius Claudius Pulcher (Paris)

David, JM (1992) Le patronat judiciaire au dernier siècle de la République romaine (Rome)

Deniaux, E (1987) “Atticus et l'Epire” in P Cabanes (ed) L'Illyrie méridionale et l'Epire dans l'Antiquité (Clermont-Ferrand): 245-254

Deniaux, E (1993) Clientèles et pouvoir à l'époque de Cicéron (Rome)

Dettenhofer, MH (1992a) Perdita Juventus. Zwischen den Generationen von Caesar und Augustus (Munich)

Dettenhofer, MH (1992b) "Zur politischen Rolle der Aristokratinnen zwischen Republik und Prinzipat" Latomus 51: 775-795 


\section{YASMINA BENFERHAT}

Dunkel, GE (2000) “Remarks on code-switching in Cicero's letters to Atticus” Museum Helveticum 57: $122-129$

Dyck, A (2008) "Rivals into partners: Hortensius and Cicero" Historia 57: 142-173

Eyben, E (1993) Restless Youth in Ancient Rome (Londres)

Fehrle, R (1983) Cato Vticensis (Darmstadt)

Förtsch, B (1935) Die politische Rolle der Frau in der römischen Republik (Stuttgart)

Grattarola, P (1990) I cesariani dalle idi di marzo alla costituzione del secondo triumvirato, (Turin)

Gruen, E (1974) The Last Generation of the Roman Republic (Berkeley)

Harders, AC (2007) "Die verwandtschaftlichen Beziehungen der Servilia, Ehefrau des L. Licinius Lucullus: Schwester oder Nichte des Cato Uticensis?” Historia 56: 453-461

Harris, W (1976) “The development of the quaestorship" Classical Quarterly 26: 92-106

Hinard, F (1985) Les proscriptions de la Rome républicaine (Rome)

Horsfall, N (1989a) Cornelius Nepos, a Selection, Including the Lives of Cato and Atticus, (Oxford)

Horsfall N (1989b) “Atticus brings back the bacon" Liverpool Classical Monthly 14: 60-62

Keaveney, A (1982) “'Young Pompey': 106-79 BC” L'Antiquité Classique 51: 111-139

Labate, M \& Narducci E (1981) "Mobilità dei modelli etici e relativismo dei valori; il 'personnaggio' di Attico" in A Giardina \& A Schiavone (edd) Società romana e produzione schiavistica (Bari) vol 3: 127-182

Latte, K (1936) “The origin of the Roman quaestorship" Transactions and Proceedings of the American Philological Association 67: 24-33

Leslie, RJ (1950) The Epicureanism of Titus Pomponius Atticus (Philadelphie)

Marshall, A (1993) “Atticus and the genealogies" Latomus 52: 307-317

Marshall, AM (1999) “Atticus and the eastern sojourn” Latomus 58: 56-68

Martin, PM (2010) “D'un Brutus à l'autre: de la construction d'un mythe de liberté à sa confusion" in $\mathrm{Cl}$ Chillet \& C Courrier (edd) Figures de l'identité - Naissance et destin des modèles communautaires dans le monde romain (Lyon): 33-49

Millar, F (1988) “Cornelius Nepos, 'Atticus' and the Roman Revolution" Greece \& Rome 35: $40-55$

Moreau, P (1989) “La relation de pseudo-filiation entre questeur et préteur. Les vicissitudes d'un modèle politique romain tiré des relations de parenté" in F Thélamon (ed) Aux sources de la puissance: sociabilité et parenté. Actes du colloque de Rouen (12-13 nov. 1987 (Rouen): 37-46

Néraudau, JP (1984) Etre enfant à Rome (Paris)

Néraudau, JP (1996) Auguste (Paris)

Ortmann, U (1988) Cicero, Brutus und Octavian. Republikaner und Caesarianer: Ihr gegenseitiges Verhältnis im Krisenjahre 44/43 vor Ch. (Bonn)

Perlwitz, O (1992) Titus Pomponius Atticus. Untersuchungen zur Person eines einflussreichen Ritters in der ausgehenden römischen Republik (Stuttgart)

Potter, FH (1934) "Political alliance by marriage" Classical J 29: 663-674 


\section{LE GOUT DES JEUNES POUSSES: ATTICUS, BRUTUS, OCTAVE}

Roddaz, JM (1984) M. Agrippa (Rome)

Roddaz, JM (2010) “L. Cornélius Balbus, l'ami de César” in Y Le Bohec (edd), État et société aux deux derniers siècles de la République romaine. Hommage à François Hinard, (Paris): 133-143

Ryan, FX (1996) "The minimum age for the quaestorship in the Late Republic" Museum Helveticum 53: 37-41

Skinner, MB (2011) Clodia Metella: The Tribune's Sister (Oxford)

Syme, R (1967) La révolution romaine (Paris) (traduction française)

Syme, R (1986) The Augustan Aristocracy (Oxford)

Tröster, M (2008) Themes, Character and Politics in Plutarch's Life of Lucullus (Stuttgart)

Van Ooteghem, J (1959) Lucius Licinius Lucullus (Bruxelles)

Van Ooteghem, J (1967) Les Caecilii Metelli de la République (Bruxelles)

Welch, K (1996) “T. Pomponius Atticus: A banker in politics?” Historia 45: 450-471

Willcock, MM (1996) Cicero: Letters of January to April 43 (Warminster)

Wiseman, TP (1987) "Legendary genealogies in late-Republican Rome" in Roman Studies, Literary and Historical (Liverpool) 